\title{
Importância do cuidado de enfermagem para melhoria da saúde e qualidade de vida
}

\section{de portadores de lesão crônica}

\author{
Importance of nursing care for improving health and quality of life of chronic injuries
}

\section{Resumo}

Indivíduos portadores de feridas crônicas carregam consigo, além de uma lesão visível na pele ou na diminuição da integralidade cutânea, um sentimento que o diminui, ocasionando a perda da saúde e qualidade de vida. Este trabalho tem como objetivo analisar a importância dos cuidados de enfermagem para saúde e qualidade de vida do portador de lesão crônica, tendo sido para tal realizado um estudo descritivo de revisão narrativa, cuja seleção de artigos se deu por meio das bases de dados LILACS, SCIELO e REDALYC. Concluímos que o enfermeiro exerce papel de destaque para a realização de uma assistência ampliada, a fim de promover o suporte adequado para amenizar as condições inadequadas e danos a qualidade de vida do portador de lesão crônica, além da necessidade de estudos para avaliação da qualidade de vida dos portadores de lesão crônica e sua relação com os cuidados de enfermagem.

Palavras-chave: Cuidados de Enfermagem; Ferimentos e Lesões; Qualidade de vida.

\begin{abstract}
Individuals with chronic wounds carry, in addition to a visible lesion on the skin or in the reduction of skin integrality, a feeling that decreases it, causing loss of health and quality of life. This work aims to analyze the importance of nursing care for health and quality of life for patients with chronic injuries, having carried out a descriptive study of narrative review, whose selection of articles was done through the LILACS databases, SCIELO and REDALYC. We conclude that the nurse plays a prominent role in carrying out expanded care, in order to promote adequate support to alleviate inadequate conditions and the damage the quality of life of patients with chronic injuries, in addition to the need for studies to assess the quality of life of patients with chronic injuries and their relationship with nursing care.
\end{abstract}

Keywords: Nursing Care; Wounds and Injuries; Quality of life.

\section{Introdução}

O primeiro e principal meio de contato, comunicante e promotor da interação do indivíduo com o meio externo é a pele o que consequentemente a torna um órgão indispensável para à vida (SOUZA et al, 2017), a presença de "lesões na pele de difícil cicatrização sem resposta ao cuidado habitual por mais de 3 meses" segundo Brasil (2013), é o que caracteriza as chamadas lesões crônicas. 
A presença de lesões crônicas tem se tornado um grande desafio terapêutico em todo o mundo, sendo considerada um problema de saúde pública que tende a se agravar com o avanço da incidência de condições que retardem a cicatrização das feridas, tais como a presença da obesidade e doenças crônicas não transmissíveis (DCNT’s), como diabetes e doenças cardiovasculares (ALMEIDA et al, 2018).

A Organização Mundial da Saúde (OMS), define qualidade de vida como a "percepção do indivíduo de sua posição na vida, no contexto da cultura e sistema de valores no qual vive e em relação aos seus objetivos, expectativas, padrões e preocupações" (WHO, 1995), o que reforça a necessidade da discussão e inclusão da qualidade de vida entre as prioridades ao se pensar e desenvolver políticas públicas de saúde.

Nesse sentido, indivíduos portadores de lesões crônicas, destacam-se como um público que carece de atenção, isso porque, estes carregam consigo além de uma lesão visível na pele ou na diminuição da integralidade cutânea, um sentimento que o diminui com relação ao outro atrelados à existência das lesões, ocasionando danos à saúde e qualidade de vida (WAIDMAN et al., 2011; BRASIL, 2013).

Souza et al. (2017) destaca que a integralidade na assistência dos serviços de saúde é um desafio da atualidade, e que, para exercitá-la, faz-se necessária adotar uma visão sistêmica dos profissionais envolvidos na atenção ante alguns fatores determinantes e condicionantes que interferem nas condições de saúde do indivíduo, dentre estes a qualidade de vida.

Diante do exposto, esse trabalho se propôs analisar a importância dos cuidados de enfermagem para saúde e qualidade de vida do portador de lesão crônica.

\section{Metodologia}

Trata-se de um estudo descritivo de revisão narrativa de literatura, cuja questão de pesquisa (PEREIRA et al., 2018) se deu na compreensão do "impacto das lesões crônicas ao indivíduo e situações que envolvem o cuidado de enfermagem para melhoria da saúde e qualidade de vida dos pacientes portadores de lesões crônicas".

A seleção dos materiais ocorreu nas seguintes bases de dados: LILACS, SCIELO, REDALYC, sendo considerados como critérios de inclusão o material de procedência nacional, textos de acesso livre, materiais publicados e indexados nos referidos bancos de dados entre os anos de 2010 a 2020, idioma português e conteúdo relacionado a questão da pesquisa.

Todos os artigos selecionados tiverem inicialmente seus resumos lidos, sendo selecionados para leitura do documento completo os artigos que contemplavam aos itens do critério de inclusão, os quais em seguida foram lidos por inteiro ((PEREIRA et al., 2018), sendo excluídos aqueles que não estavam alinhados a questão da pesquisa.

\section{Resultados e Discussão}

Ocasionalmente a presença de lesões crônicas tem como consequência alterações na aparência, causando algumas reações que diferem de um paciente para o outro como também em amigos e familiares, tais reações irão depender da capacidade de adaptação dos indivíduos, do ritmo em que ocorre e da disponibilidade de serviços (LARA et al., 2011)

É comum observarmos em portadores de lesão crônica um certo desconforto, esse desconforto vai de encontro com Almeida et al. (2018) ao afirmarem sobre as lesões que "estas geram sofrimento, dor, infecções graves, diversas comorbidades, isolamento social, depressão, comprometimento da saúde mental de forma geral, perda da mobilidade e aumento de custos".

Campoi et al. (2019) descreve entre os impactos sociais sofridos por indivíduos portadores de lesão crônica, fatores como alterações em atividades de vida diária, diminuição de mobilidade, distúrbios de sono e autoimagem, além da redução de proventos.

Tal afirmação traz consigo a necessidade de promover novas formas para possibilitar ao indivíduo portador de lesão 
além do resgate de sua integridade física e mental, também a melhoria da sua qualidade de vida, de forma que reduza assim os impactos causados em decorrência da lesão (SILVA et al., 2013).

As doenças crônicas não transmissíveis (DCNTs) estão fortemente associadas à ao estilo de vida não saudável além de possuírem múltiplas causas, podendo ser caracterizadas por um início gradual de prognóstico incerto com longa ou indefinida duração, apresentando curso clínico instável ao longo do tempo, podendo apresentar períodos de agudização e gerar diversas incapacidades (BRASIL, 2013).

Ducan et al. (2012) refere que as DCNT's foram responsáveis por 73\% das mortes gerais e 17\% das mortes precoces registradas no ano de 2017, tendo sido as doenças cardiovasculares, câncer, doença respiratória crônica e DM as quatro principais responsáveis pela maioria desses óbitos.

Segundo Borges, Nascimento Filho e Peres Júnior (2018) na abordagem clínica dos pacientes com lesões crônicas, a enfermagem deve estar atenta à existência de DCNT's como DM, HAS, doenças cardiovasculares e entre outras, além da utilização de medicamentos, presença de possíveis infecções, aspectos nutricionais e outros possíveis fatores que podem influenciar para a cronicidade da lesão, o que reforça a necessidade de um olhar técnico-humanizado por parte do profissional.

Devido possíveis comprometimentos nas dimensões biológicas, culturais e socioeconômicas ocasionadas por ulcerações e amputações resultantes do pé diabético, Pereira et al. (2017) as considera comorbidades de grande impacto na qualidade de vida de portadores de DM, reforçando a necessidade de atenção e cuidado a esse público em virtude das possíveis complicações.

Em um estudo realizado por Borges, Nascimento Filho e Pires Júnior (2018), objetivando estimar a prevalência de lesões crônicas de um município de Minas Gerais, os autores encontraram como doenças mais comuns entre os participantes a HAS e o DM, o que dado ao aumento no risco de lesão ocasionado pelas DCNT's requer atenção e cuidado por parte dos profissionais de saúde envolvidos na assistência, com intuito de evitar e/ou diminuir possíveis complicações durante o processo de cicatrização e o que acarretaria no prolongamento da permanência da lesão.

É urgente necessidade de compreender o indivíduo portador de lesão crônica como um todo, de forma a tornar o serviço a ele prestado mais empático e humanizado, para tanto, se faz necessário repensar nas formas de abordagem e assistência prestados a estes e aos que estão à sua volta, promovendo além do conhecimento técnico científico o manejo correto e atendimento integral ao indivíduo (WAIDMAN et al., 2011; CARNEIRO; SOUZA; GAMA, 2010).

O enfermeiro assume o papel importante quanto ao planejamento de ações para promoção da saúde e prevenção de doenças, cabendo a ele realizar a avaliação da lesão durante o tratamento e acompanhamento do cuidado aos indivíduos portadores de lesões, como sendo uma conduta fundamental para a escolha da terapia adequada conforme as suas características, bem como, as orientações referentes ao autocuidado em domicílio (CAMPOI et al., 2019; MEDEIROS; PERES, 2011).

A avalição integral do portador de lesão crônica por parte da enfermagem a partir da realização de exame físico, acompanhamento da evolução, curativos, escolha do melhor método para acelerar o processo de cicatrização da lesão, escolha de técnicas que propiciem um melhor retorno venoso e ações visando evitar recidiva, são de fundamental importância para o tratamento e melhoria da qualidade de vida desses indivíduos (CARNEIRO; SOUZA; GAMA, 2010; REIS et al., 2013; CAMPOI et al., 2019).

É valido lembrar que procurando reduzir agravos, o profissional de enfermagem como integrante da Estratégia de Saúde da Família (ESF) é de supra importância na assistência promovendo melhores práticas de cuidado e oferecendo às pessoas e seus familiares a oportunidade de tornarem-se protagonistas no processo de autocuidado (SOARES; HEIDEMANN, 2018) de forma que os indivíduos passem a exercer maior autonomia, refletindo na melhoria de sua qualidade de vida e saúde ao longo do processo de tratamento e recuperação das lesões. 


\section{Considerações Finais}

Diante do exposto, onde o estado de saúde e a qualidade de vida do portador de lesão crônica são afetados devido desconfortos ocasionados em decorrência da presença da lesão, o enfermeiro exerce papel de destaque para realização de uma assistência ampliada, a fim de promover o suporte adequado para amenizar das condições inadequadas e promoção do autocuidado pelo paciente, cabendo ao enfermeiro promover uma assistência à saúde de maneira humanizada e integrada para promoção da recuperação da saúde e melhoria da qualidade de vida dos indivíduos.

Destaca-se, portanto, a necessidade de estudos a fim de avaliar a qualidade de vida dos portadores de lesão crônica e sua relação com os cuidados de enfermagem, a fim de contribuir para uma abordagem crítica e ampliada com relação ao assunto, promovendo respectivamente a melhoria e promoção da saúde desses indivíduos e maior qualidade dos serviços.

\section{Referências}

ALMEIDA, W. A. et al. Fatores associados à qualidade de vida de pessoas com feridas complexas crônicas. Fund Care Online, v.10, n. 1, p. 9-16, 2018.

BORGES, E. L.; NASCIMENTO FILHO, H. M.; PIRES JÚNIOR, J. F. Prevalência de lesões crônicas de município da zona da mata mineira (Brasil). Rev Min Enferm., v. 22, n. 1, e-1143, 2018. Disponível em:〈https://cdn.publisher.gn1.link/reme.org.br/pdf/e1143.pdf〉. Acesso em: 31 jan. 2021.

BRASIL. Ministério da Saúde. Secretaria de Atenção à Saúde. Departamento de Atenção Básica. Diretrizes para o cuidado das pessoas com doenças crônicas nas redes de atenção à saúde e nas linhas de cuidado prioritárias. Brasília: Ministério da Saúde, 2013. Disponível em:<http://189.28.128.100/dab/docs/geral/documento_norteador.pdf>. Acesso em 31 jan. 2020.

CAMPOI, A.L.M. et al. Assistência de enfermagem a pacientes com feridas crônicas: um relato de experiência. REFACS (online), v. 7, n. 2 , p. 248-255, 2019. Disponível em:<https://doi.org/10.18554/refacs.v7i2.3045>. Acesso em: 31 jan. 2021.

CARNEIRO, C. M.; SOUSA, F. B.; GAMA, F. N. Tratamento de feridas: assistência de enfermagem nas unidades de atenção primária à saúde. Rev Enferm Integrada, v. 3, n. 2, p. 494-505, 2010. Disponível em:<http://files.enfermagemead.webnode.com/200000008-7e9a87f942/03-tratamento-de-ferias-assitenciade-enfermagem.pdf>. Acesso em: 31 jan. 2021.

DUCAN, B. B. et al. Doenças Crônicas Não Transmissíveis no Brasil: prioridade para enfrentamento e investigação. Rev Saúde Pública, sup. 46, p. 126-134, 2012. Disponível em:<http://www.scielo.br/pdf/rsp/v46s1/17.pdf>. Acesso em: 31 jan. 2021.

LARA, M. O. et al. Significado da ferida para portadores de úlceras crônicas. Cogitare Enferm., v. 16, n. 3, p. 471-477, 2011. Disponível em:<https://www.redalyc.org/pdf/4836/483648968011.pdf>. Acesso em: 31 jan. 2021.

MEDEIROS, V. C.; PERES, A. M. Atividades de formação do enfermeiro no âmbito da atenção básica à saúde. Texto Contexto Enferm., v. 20 , p. $27-35,2011$. Disponível em:<https://www.scielo.br/pdf/tce/v20nspe/v20nspea03.pdf>. Acesso em: 31 jan. 2021.

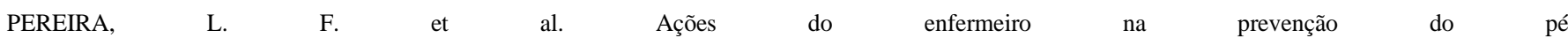
diabético: o olhar da pessoa com diabetes mellitus. Rev Fun Care Online, v. 9, n. 4, p. 1008-1014, 2017. Disponível em:<http://www.seer.unirio.br/index.php/cuidadofundamental/article/view/5702/pdf_1>. Acesso em: 31 jan. 2021.

PEREIRA, A. P. et al. Metodologia da Pesquisa Científica. Santa Maria: UFSM/NTE, 2018. Disponível em:<https://www.ufsm.br/app/uploads/sites/358/2019/02/Metodologia-da-Pesquisa-Cientifica_final.pdf>. Acesso em: 06 mar. 2021.

REIS, D. B. et al. Cuidados às pessoas com úlcera venosa: percepção dos enfermeiros da estratégia de saúde da família. Rev Min Enferm., v. 17, n. 1, p. 101106, 2013. Disponível em:〈https://cdn.publisher.gn1.link/reme.org.br/pdf/v17n1a09.pdf>. Acesso em: 31 jan. 2021.

SILVA, M. H. et al. O cotidiano do homem que convive com a úlcera venosa crônica: estudo fenomenológico. Rev. Gaúcha Enferm., v. 34, n. 3, p. 95-101, 2013. Disponível em:<https://www.scielo.br/pdf/rgenf/v34n3/a12v34n3.pdf>. Acesso em: 31 jan. 2021.

SOARES, C.F.; HEIDEMANN, I. T. S. B. Promoção da saúde e prevenção da lesão por pressão: expectativas do enfermeiro da atenção primária. Texto Contexto Enferm., v. 27, n. 2, e1630016, 2018. Disponível em:< https://www.scielo.br/pdf/tce/v27n2/0104-0707-tce-27-02-e1630016.pdf>. Acesso em: 31 jan. 2021.

SOUZA, M. R. B. O. et al. Estratégia para o cuidado em lesões de pele a partir das dificuldades relatadas por usuários da atenção básica. In: SEMANA DE ENFERMAGEM DA ESCOLA DE ENFERMAGEM DA UFRGS, 28., 2017. Porto Alegre. Anais... Porto Alegre: UFRGS, 2017, p. 10. Disponível em:<https://www.lume.ufrgs.br/bitstream/handle/10183/165741/001026091.pdf?sequence=1>. Acesso em: 31 jan. 2021.

WAIDMAN, M. A. P. et al. O cotidiano do indivíduo com ferida crônica e sua saúde mental. Texto Contexto Enferm., v. 20, n. 4, p. 691-699, 2011. Disponível em: <https://www.scielo.br/pdf/tce/v20n4/07.pdf >. Acesso em: 31 jan. 2021.

WHO - WORLD HEALTH ORGANIZATION. The World Health Organization quality of life assessment (WHOQOL): position paper from the World Health Organization. Soc Sci Med., v. 41, n. 10, p. 1403-1409, 1995. Disponível em:<https://doi.org/10.1016/0277-9536(95)00112-K>. Acesso em: 31 jan 2021. 